\title{
Research on Sensor-Less Vector Control System of PMSM Based on MRAS
}

\author{
Shengrui Zhang and Haiyan Zhang \\ Shanghai Dianji University, Shanghai 200240, China
}

\begin{abstract}
Based on the model reference adaptive system(MRAS), this paper makes a research on sensor-less vector control system of PMSM .The basic principle of MASR is analyzed, and the MRAS speed identification system of permanent magnet synchronous motor is designed. The control system model is simulated and analyzed based on MATLAB. The results show that the system tracks the motor speed accurately, and it has better dynamic performance and strong robustness.
\end{abstract}

Keywords-permanent magnet synchronous motor; model reference adaptive system; sensor-less; vector control

\section{INTRODUCTION}

The permanent magnet synchronous motor has the advantages of simple structure, small size, low loss, high torque inertia ratio, high energy density and high efficiency. In addition, with the development of power electronic technology, the research of permanent magnet synchronous motor is becoming more and more mature, and the permanent magnet synchronous motor in many areas is very popular, especially in the application of a very wide field of small and medium capacity.At present, the control strategy of permanent magnet synchronous motor is a hot research topic, the main control strategy are: open-loop constant voltage frequency ratio control, vector control, direct torque control, sliding mode control, intelligent control.

And the motion control system of permanent magnet synchronous motor needs speed feedback.In the project, the photoelectric encoder is used always, which not only increases the volume, but also increase the cost of the system, to a certain extent, reduce the reliability of the motor.So, the research on speed sensor-less technology has become a new research hotspot.At present, the motor speed estimation strategy mainly includes: the detection of inductance change method, the inverse electromotive force method, model reference adaptive method, high frequency injection method, fuzzy control method, etc..

Based on the model reference adaptive system (MRAS), this paper makes a Research on sensor-less vector control system of PMSM based on MRAS.The basic principle of MASR is analyzed, and the MRAS speed identification system of permanent magnet synchronous motor is designed.The control system model is simulated and analyzed based on MATLAB. The results show that the system tracks the motor speed accurately, and it has better dynamic performance and strong robustness.

\section{THE BASIC PRINCIPLE OF MRAS}

At present, there are many ways to estimate the rotor position and speed of the motor, and the model reference adaptive method is a new and popular method. It has the characteristics of fast adaptive speed and easy realization. Model reference adaptive method is mainly composed of three parts, which are the reference model, the adjustable model and the adaptive law. The equation with unknown parameters is selected as the reference model, and the equation with the parameters is selected as the adjustable model, like the equations of speed. The two models work at the same time, using the difference between the two models to form a suitable adaptive law, adjusting the adjustable model parameters, so as to achieve the output of the model output tracking reference model output ${ }^{[1]}$.The structure diagram of the model reference adaptive method is shown in figure 1-1:

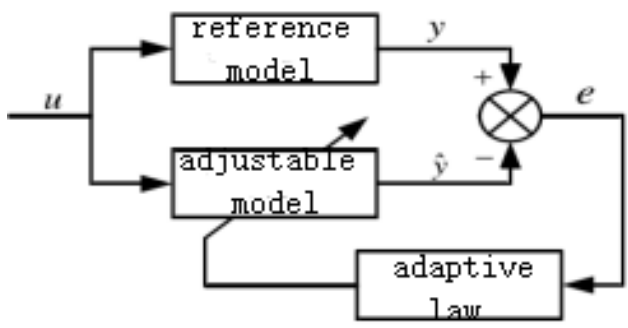

FIGURE I. MRAS STRUCTURE DIAGRAM

As shown in Figure 1-1, the reference adaptive method mainly includes: reference model, adjustable model, adaptive law three parts. The reference model and the adjustable model are excited by the same external input.Among them, $y$ and $\hat{y}$ are the state vector of the reference model and the adjustable model, the difference vector of them is $e$. Through the adaptive law, the difference vector can generate the regulating signal, which can adjust the adjustable model parameters, so that the adjustable model can approximate the reference model, and realize the goal of the output tracking reference model of the control object.

\section{Design of Mras SpeEd IdentificAtion for PMSM}

\section{A. A. The selection of reference model and adjustable model}

The stator current mathematical model of permanent magnet synchronous motor in $\mathrm{d}-\mathrm{q}$ coordinate system is: 


$$
\frac{d}{d t}\left[\begin{array}{c}
i_{d}+\frac{\psi_{f}}{L} \\
i_{q}
\end{array}\right]=\left[\begin{array}{cc}
-\frac{R_{s}}{L} & \omega_{e} \\
-\omega_{e} & -\frac{R_{s}}{L}
\end{array}\right]\left[\begin{array}{c}
i_{d}+\frac{\psi_{f}}{L} \\
i_{q}
\end{array}\right]+\frac{1}{L}\left[\begin{array}{c}
U_{d}+\frac{R_{s} \psi_{f}}{L} \\
U_{q}
\end{array}\right]
$$

It can be seen from the above formula, the stator current model of permanent magnet synchronous motor is related to the speed. Therefore, the stator current model of the motor is selected as the adjustable model, and the motor itself is a reference model, and the parallel structure is used for speed identification.

$$
i_{d}^{*}=i_{d}+\frac{\psi_{f}}{L}, i_{q}^{*}=i_{q}, U_{d}^{*}=U_{d}+\frac{R_{s} \psi_{f}}{L}, U_{q}^{*}=U_{q} .
$$

Based on the above equation (2-2)can be obtained:

$$
\frac{d}{d t}\left[\begin{array}{l}
i_{d}^{*} \\
i_{q}^{*}
\end{array}\right]=\left[\begin{array}{cc}
-\frac{R_{s}}{L} & \omega_{e} \\
-\omega_{e} & -\frac{R_{s}}{L}
\end{array}\right]\left[\begin{array}{l}
i_{d}^{*} \\
i_{q}^{*}
\end{array}\right]+\frac{1}{L}\left[\begin{array}{l}
U_{d}^{*} \\
U_{q}^{*}
\end{array}\right]
$$

Simplify above equation: $p i^{*}=A i^{*}+B U^{*}$

Final equation:

$$
\frac{d}{d t}\left[\begin{array}{l}
\hat{i}_{d} \\
\hat{i}_{q}
\end{array}\right]=\left[\begin{array}{cc}
-\frac{R_{s}}{L} & \hat{\omega}_{e} \\
-\hat{\omega}_{e} & -\frac{R_{s}}{L}
\end{array}\right]\left[\begin{array}{l}
\hat{i}_{d} \\
\hat{i}_{q}
\end{array}\right]+\frac{1}{L}\left[\begin{array}{c}
U_{d}^{*} \\
U_{q}^{*}
\end{array}\right]
$$

It can be written as: $p \hat{i}=A \hat{i}+B U^{*}$

\section{B. B. Selection of adaptive law}

The current error is defined as the different of the adjustable model and the reference model: $e=i^{*}-\hat{i}$, then:

$$
\begin{gathered}
p e=A e-W=A e+W_{1} \\
W=(\omega-\hat{\omega}) J \hat{i}, \quad W_{1}=(\hat{\omega}-\omega) J i^{*}, \quad J=\left[\begin{array}{cc}
0 & -1 \\
1 & 0
\end{array}\right]
\end{gathered}
$$

Thus the equivalent feedback system structure of MRAS is :

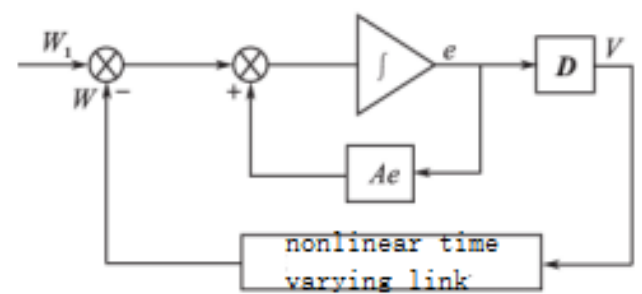

FIGURE II. MRAS EQUIVALENT NONLINEAR FEEDBACK SYSTEM
If the above system is stable, it must satisfy the Popov's inequality:

$$
\eta\left(0, t_{0}\right)=\int_{0}^{t_{o}} v^{T} W d t \geq-\gamma_{0}^{2}
$$

Proportional integral for motor speed, i.e.:

$$
\hat{\omega}=\int_{0}^{t} Y_{1}(v, t, \tau)+Y_{2}(v, t)+\hat{\omega}_{e}(0)
$$

Can get the equation:

$$
\begin{aligned}
\eta\left(0, t_{0}\right) & =\int_{0}^{t_{0}} e^{T}\left[\int_{0}^{t} Y_{1}(v, t, \tau)+\hat{\omega}_{e}(0)-\omega_{e}\right] \hat{j i} d t+\int_{0}^{t_{0}} e^{T} Y_{2}(v, t) \hat{j i} d t \\
& =\eta_{1}\left(0, t_{0}\right)+\eta_{2}\left(0, t_{0}\right)
\end{aligned}
$$

If $\eta\left(0, t_{0}\right) \geq-\gamma_{0}^{2}$, it must be met: $\eta_{1}\left(0, t_{0}\right) \geq-\gamma_{1}^{2}$, $\eta_{2}\left(0, t_{0}\right) \geq-\gamma_{2}^{2}$

In it: $\gamma_{1}^{2}, \gamma_{2}^{2}$ is any positive Co.

Can get the rotor speed estimation:

$$
\hat{\omega}_{r}=\int_{0}^{t} K_{i}\left(i_{d}^{*} \hat{i}_{q}-i_{q}^{*} \hat{i}_{d}\right) d \tau+K_{p}\left(i_{d}^{*} \hat{i}_{q}-i_{q}^{*} \hat{i}_{d}\right)+\hat{\omega}_{r}(0)
$$

It can be written as:

$$
\hat{\omega}=\left(K_{p}+\frac{K_{i}}{s}\right)\left(i_{d}^{*} \hat{i}_{q}-i_{q}^{*} \hat{i}_{d}\right)=\left(K_{p}+\frac{K_{i}}{s}\right) \varepsilon_{s}
$$

And:

$$
\varepsilon_{s}=i_{d}^{*} \hat{i}_{q}-i_{q}^{*} \hat{i}_{d}=i^{*} \times \hat{i}=i^{*} \times|\hat{i}| \sin \alpha_{s}
$$

The system block diagram can be got based on the stator current vector speed estimation:

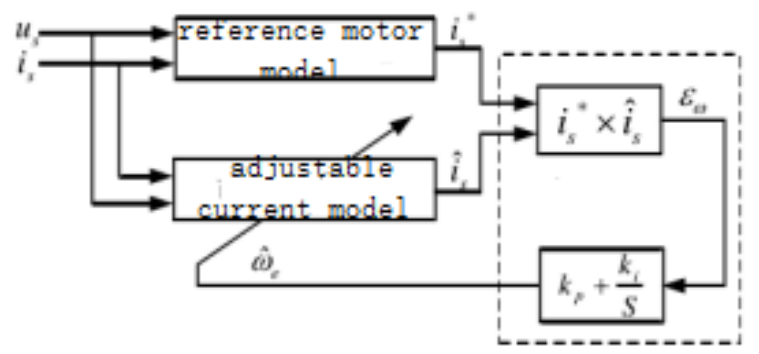

FIGURE III. THE BLOCK DIAGRAM OF THE ESTIMATION SYSTEM BASED ON THE STATOR CURRENT VECTOR

Final speed identification formula is:

$$
\hat{\omega}_{e}=k_{p}\left[i_{d} \hat{i}_{q}-i \hat{i}_{d}-\frac{\psi_{f}}{L}\left(i_{q}-\hat{i}_{q}\right)\right]+k_{i}\left[{ }_{0}^{t}\left[\hat{i}_{d} \hat{i}_{q}-i \hat{i}_{d} \frac{\psi_{f}}{L}\left(i_{q}-\hat{i}_{q}\right)\right]+\hat{\omega}_{e}(0)\right.
$$




\section{Control System Design AND Simulation ANALYSIS}

The main parts of the simulation model based on PMSM algorithm of MRAS sensorless vector control system include: coordinate transformation module, PI module, SVPWM module and MRAS speed estimation module and so on. The block diagram of sensorless vector control for permanent magnet synchronous motor is shown in figure 4 .

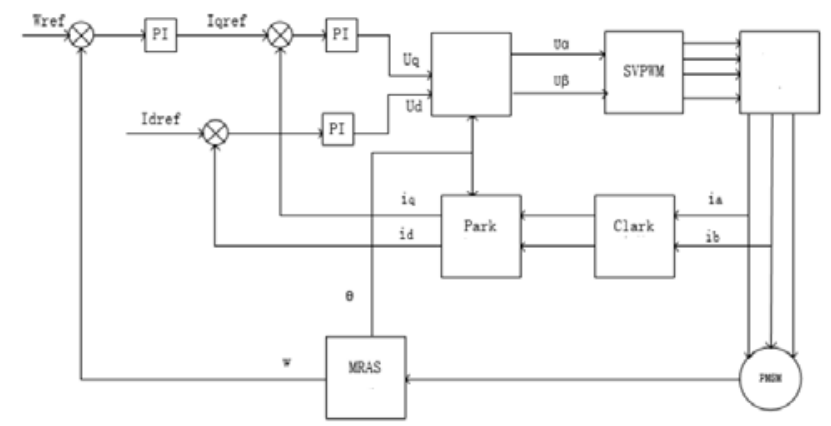

FIGURE IV. BLOCK DIAGRAM OF SENSORLESS VECTOR CONTROL FOR PERMANENT MAGNET SYNCHRONOUS MOTOR

The speed estimation module uses the MRAS algorithm, the system simulation model is shown in figure 5 .

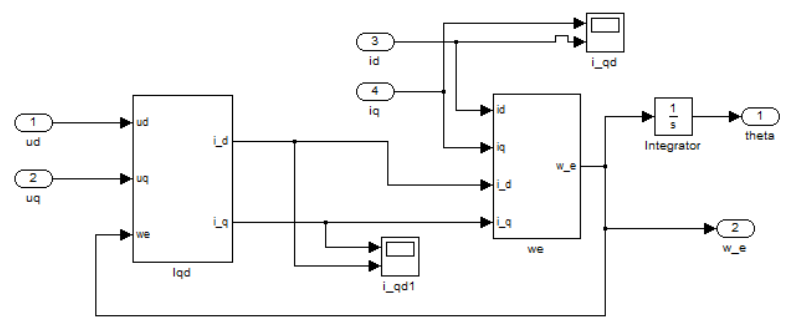

FIGURE V. SIMULATION MODEL OF MRAS SYSTEM

Based on the above foundation, the system model is built in the MTLAB/Simulink simulation environment.

The main parameters of PMSM are: $\mathrm{Rs}=2.875 \Omega$; $\mathrm{Ld}=\mathrm{Lq}=0.0085 \mathrm{H}: \psi_{f}=0.175 \mathrm{~Wb} ; \mathrm{J}=0.001 \mathrm{kgm}^{2} ; \mathrm{P}=4$. The given speed is $1500 \mathrm{r} / \mathrm{min}$,Input load torque is $0 \mathrm{~N} . \mathrm{m}$ at $\mathrm{T}=0 \mathrm{~s}$, input load torque is $1 \mathrm{~N} . \mathrm{m}$ at $\mathrm{T}=0.2 \mathrm{~s}$.

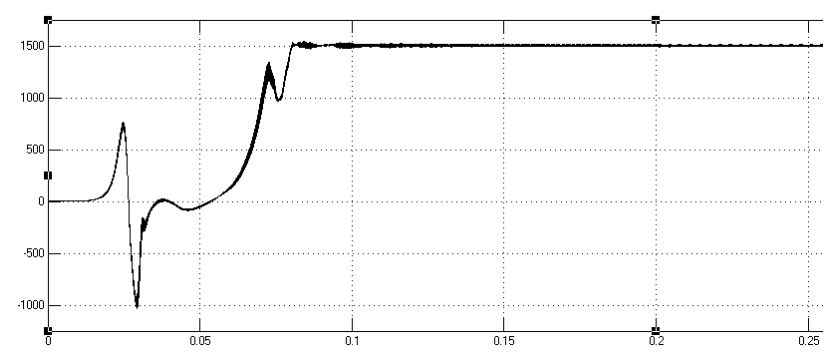

FIGURE VI. WAVEFORM OF SPEED ESTIMATION

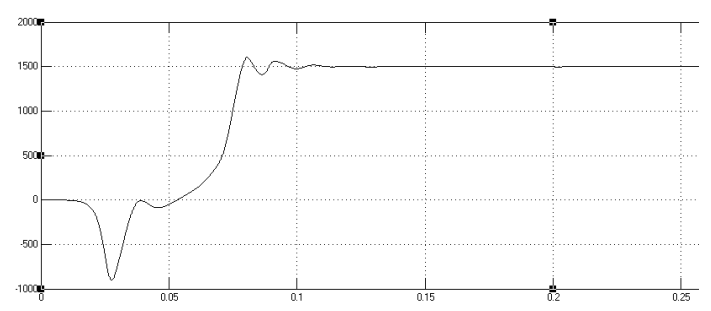

FIGURE VII. WAVEFORM OF ACTUAL SPEED

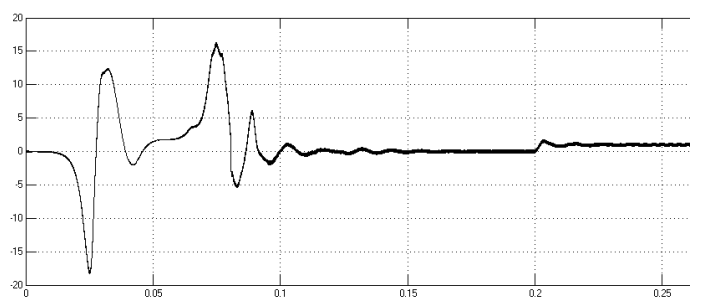

FIGURE VIII. WAVEFORM OF OUTPUT TORQUE

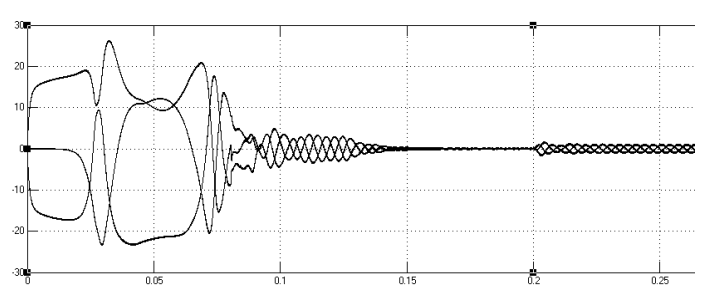

FIGURE IX. WAVEFORM OF STATOR CURRENT

Can be known from Figure 6 and Figure 7, the model reference adaptive method can be used to estimate the speed of the motor, and can realize the tracking of the actual speed of the motor.System reach a stable state after $0.1 \mathrm{~s}$, then the motor speed keeps in $1500 \mathrm{r} / \mathrm{min}$ 。

The output torque waveform is shown in Figure 8, the stator current waveform is shown in Figure 9.It can be seen that, when $t=0.2 \mathrm{~s}$, the torque is changed from 0 to $1 \mathrm{~N} . \mathrm{m}$, And at this time the output speed of the motor with the torque change also has an obvious pulse, and then quickly restored to a stable state.At the same time, the amplitude of stator current changes with the change of torque.

\section{V.CONCLUSION}

The principle of model reference adaptive algorithm is analyzed in this paper. Then the MRAS speed identification system of permanent magnet synchronous motor is designed. Finally, the system model is built in MTLAB/Simulink. And the simulation of the system is carried out under the condition of variable torque. The analysis shows that the MRAS algorithm can estimate the motor speed and track the actual speed of the motor. It avoids the use of sensors, reduces the system volume and costs.

\section{REFERENCES}

[1] Jingsong Kang,Xiangyun Zeng.Study of Position Sensorless Control of PMSM without Mechanical Sensors[J],IEEE Trans.on IA, 1992,28(6):1345-1352. 
[2] Deng Zhiquan, Chou Zhijian, and so on. Speed sensorless of permanent magnet synchronous motor based on MRAS[J]. China Journal of electrical engineering, 2007 (22):53-57.

[3] Jing Huabing, Liao Liqing. Speed sensorless control of permanent magnet synchronous motor based on model reference adaptive method[J].Electric locomotives,2007, (1): 33-36

[4] Meng Lingxin. Research on permanent magnet synchronous motor based on vector control system. Shenyang: Northeastern University, 2013:35-43.

[5] Wang Qinglong, Zhang Chongwei, Zhang Xing. Permanent magnet synchronous motor speed identification of permanent magnet synchronous motor based on variable structure model reference adaptive system [J].,2008, 9: 71-75.

[6] KIM Y S, KIM S K, KWON Y A. MRAS based sensorless control of permanent magenet synchronous motor. SICE Annual Conference in Fukui, 2003:1632-1637.

[7] Hong Naigang. MATLAB Simulation of Power Electronics and Electric Drive System[M]. Beijing : China Machine Press, 2006 : 150-180 